\title{
Cross Sectional Study of the Trend of Use of Medications and Complementary Therapy by Travelers during Flights
}

\author{
Lamya Alnaim ${ }^{1}$, Alaa AlHarthi ${ }^{1}$, Lamya AlKhalifah ${ }^{1}$, Maha AlHarthi ${ }^{1}$ \& Rawan AlMalki ${ }^{1}$ \\ ${ }^{1}$ Department of Clinical Pharmacy, College of Pharmacy, King Saud University, Riyadh, Kingdom of Saudi \\ Arabia \\ Correspondence: Department of Clinical Pharmacy, College of Pharmacy, King Saud University, Riyadh 11451, \\ Kingdom of Saudi Arabia. E-mail: lamya.alnaim@gmail.com
}

Received: August 1, 2017 Accepted: August 25, 2017 Online Published: September 6, 2017

doi:10.5539/gjhs.v9n10p85 URL: https://doi.org/10.5539/gjhs.v9n10p85

\begin{abstract}
Long distance travelling is associated with differential negative effects, collectively referred as 'travel fatigue', which results from anxiety about the journey. The study has aimed to determine the trends of using prescription drugs rather than over the counter medications and complementary alternative therapy taken by travelers. A quantitative research approach has been used and 629 travelers were recruited from Saudi Arabia. The survey questionnaire was developed by the researchers after a comprehensive literature review, and it has been modified based on a pilot study consisting of 45 people, in addition to the expert review. For a 4-month period, the survey was distributed electronically through social media, also to electronic surveys distributed to travelers at King Khalid International Airport. Travelers used either prescription and over the counter medications or Complementary alternative therapy or Non-pharmacological therapy (NPT). OTC medications such as products containing paracetamol and antihistamines were commonly used medications. About $54.6 \%$ of people reported that their choice was based on information gathered from other people's experiences, such as friends and family, followed by pharmacists. Travelers believed that medications could be used without prescription, which confirmed the extensive need of educational training related to travel and medication use.
\end{abstract}

Keywords: aerophobia, complementary alternative therapy, medications, non-pharmacological therapy, travel fatigue, travelers

\section{Introduction}

Psychological and physical health of passengers have been observed affected by the additional stress due to rapid changes in air travel (Cummings \& Worley, 2014). Travel fatigue is an extra complex summation of environmental, physiologic and psychological factors that accumulate during an individual trip (Richards, Cleland, \& Zuckerman, 2016). Passengers with particular medical conditions such as heart and lung diseases cannot tolerate the reduced oxygen level on board (Richards, Cleland, \& Zuckerman, 2016). The most common symptoms occurred during air flight is respiratory symptoms along with motion sickness. Moreover, travelers on intercontinental flights use herbal medicine, Echinacea for symptoms and development of respiratory diseases. Many special services have been offered by major carriers with increased attention to passengers with disabilities. Health risks associated with air travel can be reduced, when the traveler plans carefully and takes precautions throughout the flight (Weinberg, Weinberg, \& Maloney, 2012).

Some travelers experience aerophobia (an abnormal and persistent fear of flying) or a similar anxiety disorder. However, all flights, short or long haul, impose stress on passengers. Preflight stress includes carrying baggage, walking long distances, getting to the gate on time, and flights being delayed. In-flight stress includes acceleration, vibration (including turbulence), noise, lowered barometric pressure, temperature and humidity variation and fatigue, among others (Thibeault \& Evans, 2015; Waterhouse, Reilly, \& Edwards, 2004).

This study aimed at identifying the trends of Rx (prescription) and OTC (Over the counter) medication and CAM (Complementary alternative medicine) used by travelers in Riyadh, Saudi Arabia during long distance flights. The objectives of the study were to define the most common medications and CAM's used by travelers during long distance flights. The study further identified the source of information that travelers used regarding these medications and CAMs. The study significantly contributed to assess the perception of travelers regarding the use of these medications without a prescription and identify the adverse effects of the use of these medications and 


\section{CAMs.}

The study has reported that travelers are always looking for the solution to these stressors. They commonly use Rx medications, OTC medications or CAM. Pharmacists are obligated to know the trend of medications and complementary therapy being used by travelers to find out the method of use, educate them how to use it correctly and suggest other non-pharmacological solutions that should be taken into consideration. Interest in this topic originated from the fact that there are no studies to describe the trend of using the medications and complement therapy during flights in Saudi Arabia. The aim of the study has effectively contributed towards the field of environmental and public health concerns. The results may enhance the travel practices of the travelers and provoke the pharmacists and other health professionals to promote positive and influential strategies for the travelers.

\section{Materials and Methods}

A quantitative research design has been employed to retrieve the outcomes of the study. A survey was conducted based on 629 travelers from Saudi Arabia including adults, children, both Saudi nationals, and non-Saudis. A questionnaire was developed for the data collection process for the quantitative analysis. The questionnaire was used initially for pilot survey to examine the reliability and validity of the factors included in the questionnaire. The pilot testing was based on the sample consisting of 45 people. The effective outcomes of the pilot test were evaluated through Statistical Package of Social Sciences (SPSS) version 20.0. Cronbach's Alpha test was applied on the developed questionnaire, which confirmed the validity and reliability of the data. The survey was conducted for 4-months, between December 2015 and March 2016. A total of 650 questionnaires were sent through online access to collect the data from which 629 were received with complete information. Incomplete questionnaires were excluded for the analysis. The survey instrument was distributed to the participants through social media and electronic surveys were distributed to the travelers at King Khalid International Airport. Statistical Package of Social Sciences (SPSS) version 20.0 was used for the data analysis. Descriptive statistics was evaluated to describe the sample observations and a Pearson correlation was used to assess the relationship between study variables, which included age, sex, academic qualifications and participant's viewpoints, experiences and actions. An informed consent form was provided along with the questionnaire to the participants to ensure the privacy and confidentiality of the data obtained from them regarding their experiences and practices.

\section{Results}

The age distribution of the study sample has been shown in Table 1. The age group from 21 years to 40 years was the most predominant in the study sample (54\%), followed by 13 to 21 years $(28.9 \%)$.

Table 1. Age Distribution of Study Sample

\begin{tabular}{ll}
\hline Age & Percent \\
\hline From one month to 12 month & 0.8 \\
Older than a year to 12 years & 3.0 \\
From 13 years to 21 years & 28.9 \\
From 21 years to 40 years & 54.8 \\
From 40 years to 61 years & 11.9 \\
Older than 61 years & 0.5 \\
Total & 100.0 \\
\hline
\end{tabular}

The proportion of males and females that participated in the study is shown in Figure 1A. The majority of the participants were females (77.1\%). The results observed majority of Saudi participants $(90.6 \%)$; while, $9.4 \%$ were Non-Saudis. Regarding the academic qualification, $59.1 \%$ of the study sample held a bachelor's degree, followed by secondary school qualification (24.3\%), while the rest varied between postgraduate and diploma (16.6\%).

Almost eighty-two percent (81.4\%) of the study sample claimed that they did not suffer from any chronic disease, while $18.6 \%$ disclosed that they suffered from chronic diseases such as asthma, diabetes, hypertension, hypothyroidism, eczema and colon diseases. The most frequently reported diseases were asthma, diabetes, and hypertension, respectively.

The frequency of 'travel fatigue' reported by study participants is shown in Figure 1B. Travel fatigue can be 
defined as a common perspective, which refers to the occurrence of fatigue due to enhanced level of travelling or physical exercise. The majority of participants have suffered fatigue or exhaustion during flights $(77.4 \%)$, while the remainder did not suffer from these issues $(22.6 \%)$. A portion of travelers indicated suffering from anxiety or phobia related to airplane travel (14.5\%).

Travelers who did not suffer from fatigue or sleep disturbances were Group 1 Travelers, travelers who suffered from fatigue or sleep disturbances but did not try to remedy the problem were Group 2 Travelers. Travelers, who suffered from fatigue or sleep disturbances and used medications or complementary therapy as a solution (Group 3 Travelers). Group 1 travelers made up the majority of study sample at $77.4 \%$. Group 2 Travelers accounted for approximately $2 \%$ of the study sample. Group 3 Travelers accounted for approximately $20.6 \%$. Solutions used by the study sample are shown in Figure 1C. The Solutions Group 3 Travelers used varied between three categories; Prescription (Rx) and over the counter (OTC) medications, Complementary alternative therapy (CAM), and Non-pharmacological therapy (NPT).
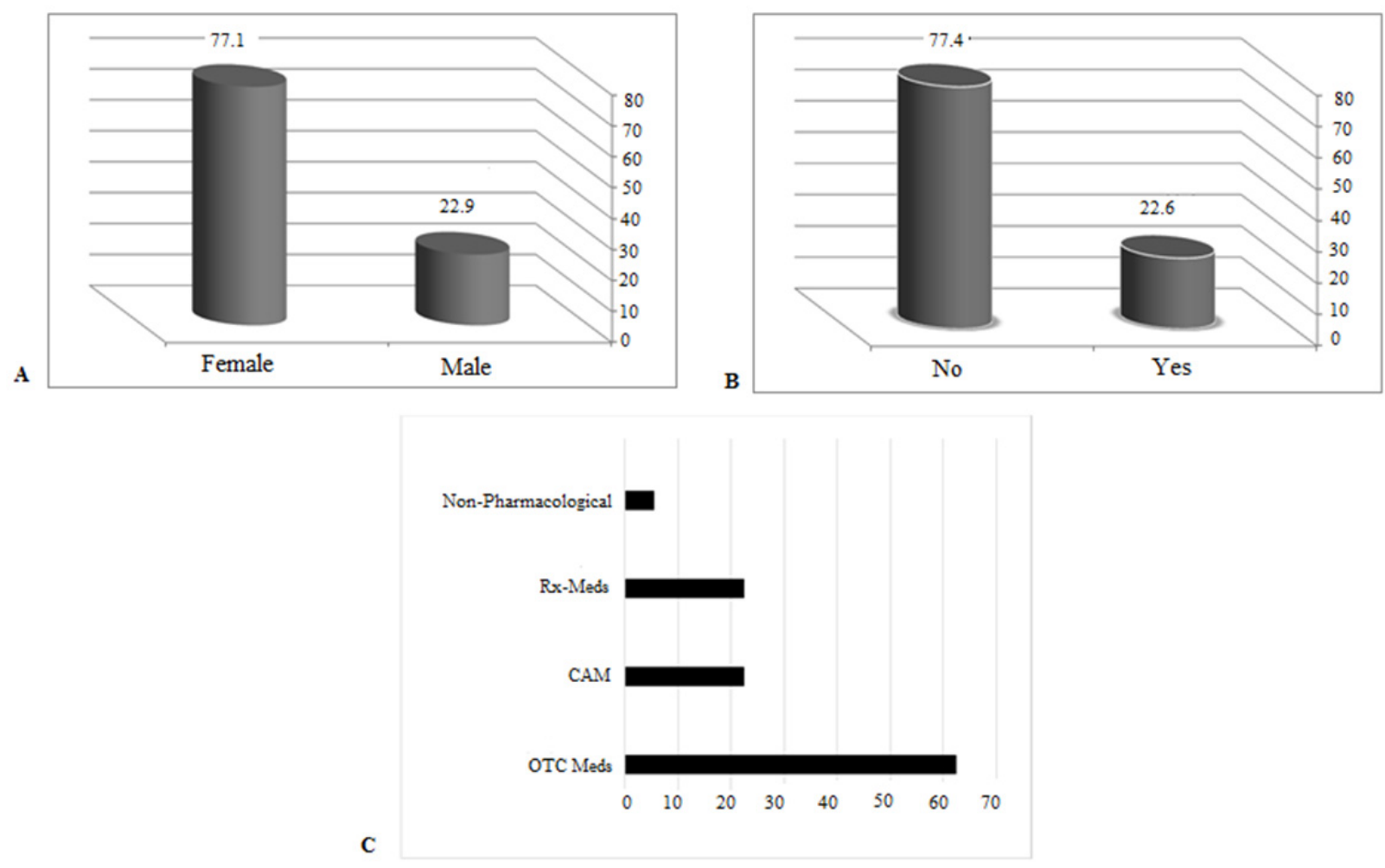

Figure 1. (A) The Distribution of the Study Sample According to the Gender; (B) Frequency of Travel Fatigue Reported by Study Participants; (C) Solutions used by the Study Sample

The OTC medications made up the majority of solutions used by the study population at $61.5 \%$, followed by CAM at $22 \%$. The most common solutions used by the study sample are shown in Table 2 . Among the solutions used by the participants, paracetamol and ibuprofen products were the most predominant OTC medications used (39.2\%) followed by antihistamines and paracetamol/antihistamines combination products $(11.5 \%$ and $10.76 \%$ respectively). Other medications reported were hyoscine, propranolol, and aspirin in addition to sage, thyme, and caraway. Non-pharmacological treatments varied between sleeping, chewing gum for relieving nausea, relaxation and breathing exercises $(5 \%)$. About $4.6 \%$ of participants admitted they do not remember the name of medication they used. 
Table 2. Most Common Solutions Used by the Study Sample

\begin{tabular}{ll}
\hline Item & Percent $\%$ \\
\hline Paracetamol /Ibuprofen & $39.2 \%$ \\
Antihistamines & $11.5 \%$ \\
Paracetamol/Antihistamine Combination products & $10.76 \%$ \\
Antidepressants & $2.3 \%$ \\
Benzodiazepine & $2.3 \%$ \\
Melatonin \& Serotonin products & $3.8 \%$ \\
Mint & $8.5 \%$ \\
Caffeine & $3.8 \%$ \\
Anise & $3 \%$ \\
Chamomile & $3 \%$ \\
Non-Pharmacological & $5 \%$ \\
\hline
\end{tabular}

The frequency of using these solutions in repeated flights, as well as in a single flight is shown in Table 3 . Approximately, one-third of the study sample is looking for solutions in flights over 6 hours. And about $26.15 \%$ only have a single experience. Approximately $85.4 \%$ of the study sample used their solutions only once per flight. The sources of information participants used to identify their solutions were other people's experiences, such as friends and family in more than half of the sample (54.7\%). Pharmacists were the second most common source of information (34.6\%). Only $15.4 \%$ used a medication based on a valid prescription. Participant's attitude toward their experiences is shown in Table 3 . Approximately $74.6 \%$ of participants revealed that they used these solutions as they believed they were effective. Alternatively, $16.9 \%$ confirmed that they would not repeat their use because of lack of effectiveness.

Table 3. Time and frequency of use of solution by participants and Participants Attitude towards Their Experience

\begin{tabular}{|c|c|}
\hline Question & Percent \\
\hline \multicolumn{2}{|l|}{ Use in all flights } \\
\hline In every flight & $16.15 \%$ \\
\hline In flight over 3 hours only & $16.15 \%$ \\
\hline In flight over 6 hours only & $41.5 \%$ \\
\hline Only once & $26.15 \%$ \\
\hline \multicolumn{2}{|l|}{ Times per flight } \\
\hline One time & $85.4 \%$ \\
\hline Two times & $7.7 \%$ \\
\hline Three times & $1.5 \%$ \\
\hline All the time & $2.3 \%$ \\
\hline Five times & $0.4 \%$ \\
\hline Before the flight only & $0.4 \%$ \\
\hline When necessary & $0.4 \%$ \\
\hline Participants attitude towards their experience & Percent $(\%)$ \\
\hline Repeat, effective & 74.6 \\
\hline Not-repeat, Not-effective & 16.9 \\
\hline Not-repeat, Side effects & 4.6 \\
\hline Not-repeat, Future risk & 3.8 \\
\hline
\end{tabular}


The frequency of side effects that the participants experienced was reported at $20 \%$, while the most common side effect observed was nausea and headache(13.6\%), followed by shortness of breath and an increase in the heart rate (11.4\%). When asked about travelers' perception towards the use of sleeping aid medication without the prescription, $63.4 \%$ of the surveyed population disagreed and $36.6 \%$ agreed that medications could be used without the prescription. Pearson correlation coefficient was used to estimate the relationship between study variables, such as age, sex, academic qualifications and participant's viewpoints, experiences and actions.

There was no significant correlation between the viewpoint of participants regarding the solutions they used to relieve fatigue and their academic qualification. The symptoms felt after they used the drug or herb, or the fatigue level they were experiencing were observed. There were also no statistically significant differences between the source of information and age, sex or academic qualification.

Prevalence of anxiety or phobia was not correlated with sex or age group of participants. Furthermore, there was no significant correlation between participants suffering from anxiety or phobia and the solutions that are used to relieve fatigue. Additionally, there was no correlation between participants' opinions about requiring prescriptions and their age or academic qualifications. Table 4 shows the correlation of relationships between study variables.

Table 4. The Correlation of Relationships between Study Variables

\begin{tabular}{|c|c|c|c|c|c|}
\hline & $\begin{array}{l}\text { Did you feel any of the } \\
\text { following diseases after } \\
\text { using the drug or herb }\end{array}$ & $\begin{array}{l}\text { Academic } \\
\text { qualification }\end{array}$ & Age & $\begin{array}{l}\text { Do you suffer } \\
\text { from anxiety or } \\
\text { phobia? }\end{array}$ & $\begin{array}{l}\text { Fatigue } \\
\text { level }\end{array}$ \\
\hline $\begin{array}{l}\text { Solutions used to relieve fatigue or } \\
\text { minimize it }\end{array}$ & $.152 *$ & $-.070^{*}$ & NA & $-.189 *$ & $.007 *$ \\
\hline $\begin{array}{l}\text { From your point of view, the use of } \\
\text { sedative drugs during the flights do } \\
\text { not require a prescription }\end{array}$ & NA & $.008 *$ & $-.009 *$ & NA & NA \\
\hline Methods of getting such solutions & NA & $.054^{*}$ & $-.001 *$ & NA & NA \\
\hline
\end{tabular}

*Pearson correlation coefficient.

\section{Discussion}

The study has demonstrated that long distance is always associated with various transient negative effects, collectively referred to as 'travel fatigue'. It may results from anxiety about the journey, change of an individual's daily routine and dehydration resulting from time spent in the dry air of the aircraft cabin. Sleep deprivation and its associated effects are significant components of travel fatigue. Attempts to promote sleep by the use of prescription (Rx), over the counter (OTC) medications or complementary alternative therapy (CAM) are common.

Since the end of the Second World War, international air travel has become so frequent that many people travel on long-distance routes every day. 'Travel fatigue' is a condition frequently experienced by travelers on any long-distance journey and is not associated specifically with air travel across time zones (Simmons, McGrane, \& Wedmore, 2015). 'Travel fatigue' has been extensively studied in sports medicine, and defined as a more complex summation of physiological, psychological, and environmental factors that accrue during an individual trip (Samuels, 2012).

Anxiety or phobia of flying was reported in $14.5 \%$ of participants. Global estimates and diagnostic criteria vary. For instance, in 2008, a survey of 7076 participants randomly selected Dutch adults found a lifetime prevalence of $2.5 \%$ utilizing the DSM-III criteria for specific/simple phobia (Depla et al., 2008). In the US, a survey of 43093 adults utilizing criteria of excessive fear resulting in restricted activity, with a lifetime prevalence of $2.9 \%$ was observed (Stinson et al., 2007).

'Travel fatigue' may be due to many factors, such as the cramped seats, altered feeding schedule, alcohol consumption, poor air quality, and inability to sleep (Srinivasan et al., 2010). Several strategies have been studied to help reduce or resolve travel fatigue. These strategies include pharmacological treatments, circadian interventions and supplementary clinical therapies (Samuels, 2012). The majority of studies on 'travel fatigue' were conducted on athletes. Pharmacological strategies to combat fatigue include melatonin, short and medium acting hypnotics and caffeine (Samuels, 2012).

Melatonin has been extensively studied in sleep disturbances in general and those related to jet lag. The efficacy of 
melatonin and its analogues is subjected to a high degree of inter-individual and intra-individual variability, as described in several meta-analysis and reviews (Samuels, 2012; Depla et al., 2008). The American Academy of Sleep Medicine recommends the timed use of melatonin to hasten adaptation (Sack et al., 2007).

Non-pharmacological interventions include light therapy for circadian rhythm shifting, napping and timing of feedings (Samuels, 2012). According to the obtained results, travelers also used pharmacological and non-pharmacological strategies to cope with 'travel fatigue'. Melatonin made up less than $4 \%$ of the strategies used. Acetaminophen or Ibuprofen alone, or in combination with first generation H1 blockers, were the most frequent pharmacological agents used. These agents, although effective analgesics and fairly safe to the general population do not have a specific effect on fatigue.

The use of pharmacological and non-pharmacological strategies related to the source of information travelers depend on their pre-travel advice. Medications, whether Rx or OTC, made up of $66 \%$ of the strategies used. Non-pharmacological options made up only $5 \%$ of the strategies.

This study has highlighted areas of concerns; firstly, of the travelers that used prescription medications, which evaluated that only $15.4 \%$ obtained these medications as a result of a valid prescription. Benzodiazepine, for example, can produce unexpected adverse effects that can be unsafe during air travel such as hallucinations which might disturb the flight and over sleepiness which hinder the traveler from completing post arrival steps. Secondly, up to $36 \%$ of the study sample believed a prescription is not needed to obtain such medications before travel. Thirdly, many of the pharmacological strategies used have no role in the prevention of 'travel fatigue', have not helped the patient and had the potential to cause unnecessary harm. Lastly, more than half of study participants depended on lay sources for the information regarding treatment and prevention of 'travel fatigue'.

Opportunities have been increased for patients with COPD and other respiratory diseases that commonly require oxygen for travel. Airline personnel have adjusted the needs of passengers with respiratory diseases. Two major factors have been found that have a greater effect on passengers with COPD (Davies, 2016).

It has been indicated that special considerations have been offered to the passengers of anaemia. These patients are provided with hemoglobin below $7.5 \mathrm{gm} / \mathrm{dl}$ because it decreases the tolerance of hypoxia (Palakanis, 2012). However, oxygen was also considered by some passengers as an alternative treatment. A lower hemoglobin level at cabin cruising altitude has been tolerated by passengers with chronic renal failure and other conditions predisposing to anemia (Lim et al., 2014).

Community pharmacists may become involved in travel medicine services, due to their ease of accessibility with many convenient locations, long hours of operation. There are many models for pharmacist's role in travel medicine (Seed et al., 2011). Also, clinics in community pharmacies can provide phone consultation and individualized appointments for travel advice. Even outside such structured programs, a pharmacist can provide travel advice service in their pharmacies, especially when prompted by a consumer wishing to buy medications for 'travel fatigue' or to help sleep on a flight.

It has been recommended that pharmacists should dispense prescription medication with a valid prescription; make the safe recommendation from over the counter products based on the consumer's health history. A recommendation regarding immunizations and preventive measures for travel related disease must be made. Providing these services within their daily routine will establish a stronger role for the pharmacist as a first line healthcare provider in this area and will increase the trust in their services. This will assist in the development of specialized services that pharmacists can provide such as in the form of travel clinics.

\section{Conclusion}

The trend of medication and complementary therapy used during flights by this study sample predominantly consists of products containing Paracetamol. Unfortunately, a significant proportion of travelers still receive their drug information from other people's experiences such as friends and family and not from a valid source such as a pharmacist. A substantial portion of the study population also believed that medications could be used without a prescription. There is a burden on government bodies of the Saudi Food and Drug Authority (SFDA) to educate the public regarding prescription medications and travel, and to enforce the role of the pharmacist in dispensing medication in the community setting. Travel health advice is a great opportunity for pharmacists to establish their role as an easily accessible competent health care provider. Also, results of this study have shown that there are an extensive need and enormous scope for education related to travel and medication use.

\section{Acknowledgments}

The author is very thankful to all the associated personnel in any reference that contributed in/for the purpose of 
this research. The author declares no conflict of interest.

\section{Competing Interests Statement}

The authors declare that there are no competing or potential conflicts of interest.

\section{References}

Cummings, T. G., \& Worley, C. G. (2014). Organization development and change. Cengage learning. https://doi.org/10.1002/9780470753408.ch2

Davies, G. (2016). Respiratory disease. Ernsting's Aviation and Space Medicine 5E. 427.

Depla, M. F., Margreet, L., van Balkom, A. J., \& de Graaf, R. (2008). Specific fears and phobias in the general population: results from the Netherlands Mental Health Survey and Incidence Study (NEMESIS). Social psychiatry and psychiatric epidemiology, 43(3), 200-208. https://doi.org/10.1007/s00127-007-0291-z

Lim, H. Y., Huang, G. K. L., Torresi, J., \& Johnson, D. (2014). Red Herring in Returned Traveler: Drug Reaction With Eosinophilia and Systemic Symptom (DRESS) Syndrome Mimicking Sepsis. Journal of travel medicine, 21(6), 425-428. https://doi.org/10.1111/jtm.12152

Palakanis, K. C. (2012). Clinical Practice Guidelines for Primary Care Providers' Evaluation of Patient Fitness to Fly.

Richards, P., Cleland, J., \& Zuckerman, J. (2016). Psychological factors relating to physical health issues: how physical factors in aviation and travel affect psychological functioning. Aviation Mental Health: Psychological Implications for Air Transportation, 27.

Sack, R. L., Auckley, D., Auger, R. R., Carskadon, M. A., Wright Jr, K. P., Vitiello, M. V., \& Zhdanova, I. V. (2007). Circadian rhythm sleep disorders: part I, basic principles, shift work and jet lag disorders. Sleep, 30(11), 1460-1483. https://doi.org/10.1093/sleep/30.11.1460

Samuels, C. H. (2012). Jet lag and travel fatigue: a comprehensive management plan for sport medicine physicians and high-performance support teams. Clinical Journal of Sport Medicine, 22(3), 268-273. https://doi.org/10.1097/JSM.0b013e31824d2eeb

Seed, S. M., Spooner, L. M., O'connor, K., \& Abraham, G. M. (2011). A multidisciplinary approach in travel medicine: the pharmacist perspective. Journal of travel medicine, 18, 352-4. https://doi.org/10.1111/j.1708-8305.2011.00544.x

Simmons, E., McGrane, O., \& Wedmore, I. (2015). Jet lag modification. Current sports medicine reports, 14, 123-8. https://doi.org/10.1249/JSR.000000000000133

Srinivasan, V., Singh, J., Pandi-Perumal, S. R., Brown, G. M., Spence, D. W., \& Cardinali, D. P. (2010). Jet lag, circadian rhythm sleep disturbances, and depression: the role of melatonin and its analogs. Advances in therapy, 27(11), 796-813. https://doi.org/10.1007/s12325-010-0065-y

Stinson, F. S., Dawson, D. A., Chou, S. P., Smith, S., Goldstein, R. B., Ruan, W. J., \& Grant, B. F. (2007). The epidemiology of DSM-IV specific phobia in the USA: results from the National Epidemiologic Survey on Alcohol and Related Conditions. Psychological medicine, 37(7), 1047-1059. https://doi.org/10.1017/S0033291707000086

Thibeault, C., \& Evans, A. D. (2015). AsMA medical guidelines for air travel: stresses of flight. Aerospace medicine and human performance, 86(5), 486-487. https://doi.org/10.3357/AMHP.4225.2015

Waterhouse, J., Reilly, T., \& Edwards, B. (2004). The stress of travel. J Sport Sci., 22, 946-66. https://doi.org/10.1080/02640410400000264

Weinberg, N., Weinberg, M., \& Maloney, S. (2012). Traveling safely with infants and children. CDC health information for international travel.

\section{Copyrights}

Copyright for this article is retained by the author(s), with first publication rights granted to the journal.

This is an open-access article distributed under the terms and conditions of the Creative Commons Attribution license (http://creativecommons.org/licenses/by/4.0/). 